\title{
COMPORTAMIENTO DE LA INFILTRACIÓN EN UN OXISOL
}

\section{BEHAVIOR OF THE INFILTRATION ON AN OXISOL}

\author{
Helber M. Orjuela-Mattaํㅜ, Yolanda Rubiano Sanabria² ${ }^{2}$ Jesús Hernán Camacho-Tamayo³
}

\begin{abstract}
${ }^{1}$ Ingeniero Agrícola, M.Sc. Universidad Nacional de Colombia. Facultad de Ingeniería, Programa de Ingeniería Agrícola. Cra. 45 No. 45 - 03, Bogotá, Colombia. E-mail: hmorjuelam@gmail.com² Dra. Sc. Agropecuarias Suelos, Profesora Asistente. Universidad Nacional de Colombia. Facultad de Agronomía. Cra. 45 No. 45 - 03, Bogotá, Colombia. E-mail: yrubianos@unal. edu.co ${ }^{3}$ Ingeniero Agrícola, M.Sc. Profesor Asistente. Universidad Nacional de Colombia. Facultad de Ingeniería, Programa de Ingeniería Agrícola. Cra. 45 No. 45 - 03, Bogotá, Colombia. E-mail: jhcamachot@unal.edu.co
\end{abstract}

Rev. U.D.C.A Act. \& Div. Cient. 13 (2): 31-39, 2010

\section{RESUMEN}

El estudio de las técnicas de infiltración de agua en el suelo permite entender los procesos hidrológicos, la degradación de suelos y el transporte de solutos, siendo un soporte significativo, para minimizar los métodos de escorrentía superficial. El objetivo del presente trabajo fue evaluar el comportamiento de la infiltración, a través de los modelos de Kostiakov, KostiakovLewis y Philip. El trabajo fue realizado en el municipio de Puerto López (Meta-Colombia). La metodología incluyó la ejecución de 64 pruebas de infiltración, usando anillos infiltrómetros dobles, en una malla rectangular de muestreo de $52 \mathrm{~m} \times 45 \mathrm{~m}$. En cada punto de la malla, se tomaron muestras de suelo, a $10 \mathrm{~cm}$ de profundidad, para determinar textura, humedad volumétrica y densidad aparente. Con las curvas de infiltración experimental, se realizaron ajustes a los modelos empíricos para cada punto. Para el análisis de datos, se implementó la estadística descriptiva, con el fin de conocer el comportamiento y la dispersión de las propiedades del suelo. De la misma manera, se establecieron las correlaciones entre las propiedades del suelo y los parámetros de cada modelo. Los datos experimentales tuvieron un mejor ajuste al modelo de Kostiakov-Lewis, seguido del modelo de Kostiakov, con un $\mathrm{R}^{2}$ de 0,99 y 0,98, respectivamente. La propiedad que mayor incidencia presentó en la infiltración fue la densidad aparente, observándose una correlación inversa con la mayoría de los parámetros de los modelos. La variabilidad de la infiltración indica la conveniencia de identificar zonas de manejo, para evitar déficit o excesos de agua, que pueden someter el cultivo a estrés hídrico o favorecer procesos de escorrentía superficial.

\section{SUMMARY}

The study of water infiltration processes into the soil helps to understand hydrological processes, soil degradation and solutes transport, with significant support to minimize runoff processes. The aim of this study was to evaluate the infiltration behaviour through Kostiakov, Kostiakov-Lewis and Philip models. This research was carried out at the Puerto López municipality (Meta-Colombia). The methodology included the implementation of 64 infiltration tests, using double ring infiltrometer, located in a rectangular grid sampling of $52 \mathrm{~m}$ by $45 \mathrm{~m}$. At each grid point soil samples were taken at $10 \mathrm{~cm}$ depth, to determine texture, bulk density and volumetric moisture. With the experimental infiltration curves adjustments were made to the empirical models for each point. For data analysis descriptive statistics were implemented in order to understand the behaviour and dispersion of soil properties. Correlations between soil properties and parameters of the estimated models were also established. The experimental data were better fit the Kostiakov-Lewis model, following the model of Kostiakov, with an $\mathrm{R}^{2}$ of 0.99 and 0.98 , respectively. The property of higher incidence over the infiltration was the bulk density, showing an inverse correlation with most of the parameters. The infiltration variability indicates the desirability of identifying management zones, to avoid a deficit or excess water, which can carry the crop to water stress or contribute to runoff processes.

Key words: Physical properties, infiltration models, water movement.

Palabras clave: Propiedades físicas, modelos de infiltración, movimiento del agua. 


\section{INTRODUCCIÓN}

El orden de suelos predominantes en el trópico son los Oxisoles, con un área de 9,61 millones de $\mathrm{km}^{2}$, equivalente al 24,52\% de la superficie de la zona tórrida (USDA, 1999). Colombia, por su ubicación cercana al eje del Ecuador, presenta áreas importantes de este orden de suelos, principalmente, localizadas en los Llanos Orientales. Desde el punto de vista físico son suelos de una alta fragilidad, fácilmente degradados por erosión hídrica, dado que se encuentran en una zona que está bajo un régimen de lluvias monomodal, donde se registran meses con alta intensidad pluviométrica, que conjuntamente con procesos naturales de adensamiento y bajos niveles de porosidad (Ramírez-López et al. 2008), manifiestan limitantes en los procesos naturales de infiltración y de drenaje.

La comprensión de los procesos de infiltración de agua en el suelo permite entender los procesos hidrológicos, la degradación de los suelos y el transporte de solutos, así como servir de soporte para minimizar los procesos de escorrentía superficial. Adicionalmente, la infiltración es uno de los parámetros básicos y más importantes para el diseño e implantación de sistemas de irrigación, mediante aspersión, buscando un uso eficiente y adecuado del agua (Chowdary et al. 2006; Machiwal et al. 2006; Silva, 2007).

La infiltración es una propiedad hidrodinámica, que considera el movimiento vertical del agua en el suelo, estrechamente relacionada con los procesos de capilaridad y de las fuerzas asociadas con la adhesión y la cohesión de las partículas del suelo (Forero, 2000). Depende de las características intrínsecas del medio, como el contenido de materia orgánica, la porosidad, la textura, la densidad aparente y el contenido de agua, entre otros (Chowdary et al. 2006; Diamond \& Shanley, 2003; Rodríguez-Vásquez et al. 2008).

Desde hace varias décadas, los procesos de infiltración han tomado importancia, conduciendo al desarrollo de diferentes modelos que estiman la infiltración, como una función del tiempo o de algunos parámetros físicos (Green \& Ampt, 1911; Kostiakov, 1932; Horton, 1940; Philip, 1957). Haverkamp et al. (1998) encontraron que estos modelos pueden arrojar resultados no representativos, si se ignoran el contenido de agua y la variabilidad espacial de las propiedades del suelo.

En general, la tasa de infiltración varía entre puntos cercanos, como resultado de la variabilidad de las propiedades del suelo, mostrando también diferencias de los parámetros estimados, para un mismo modelo, incluso, en la misma unidad de suelo (Loague E Gander, 1990; Machiwal et al. 2006; RodríguezVásquez et al. 2008). Por esto, el objetivo del presente trabajo fue evaluar el comportamiento de la infiltración, a través de los modelos de Kostiakov, Kostiakov-Lewis y Philip, en una misma unidad de suelo.

\section{MATERIALES Y MÉTODOS}

Caracterización del área de estudio: El presente estudio fue realizado en la Estación Experimental Taluma, de CORPOICA, ubicada en el municipio de Puerto López (Meta), en las coordenadas $4^{\circ} 22^{\prime} 38,50^{\prime \prime}$ de latitud norte y $72^{\circ} 13^{\prime} 24,53^{\prime \prime}$ de longitud oeste, a una altura de 156 m.s.n.m. El suelo predominante en la zona pertenece a la Familia franco fina de Typic Haplustox, con texturas entre franco limosa y franco arcillosa y pendiente ligeramente inclinada $(<5 \%)$ y dedicado a ganadería extensiva en praderas nativas (Trachypogum vestitas). La precipitación de la zona presenta un régimen monomodal, con una media anual de $2375 \mathrm{~mm}$, concentrada entre abril a noviembre, temperatura media de $27^{\circ} \mathrm{C}$ y humedad relativa de $75 \%$ (Jaimes et al. 2003).

Toma de datos: Las pruebas de infiltración, se realizaron empleando anillos infiltrómetros dobles, en una malla rectangular, conformada por 64 puntos, a una distancia perpendicular de $52 \times 45 \mathrm{~m}$ entre puntos, realizando lecturas por un periodo de tres horas para cada prueba, en marzo de 2008. El tiempo de medición fue seleccionado teniendo en cuenta que se trata de la época seca, donde las condiciones de humedad del suelo son relativamente homogéneas y, de acuerdo con Jaimes et al. (2003), el contenido de agua en el suelo era cercano del punto de marchitez permanente, con un contenido inferior al $14 \%$, para todos los puntos de muestreo. Además, marzo se caracteriza por ser un mes con ausencia de lluvias en la zona, lo cual, garantizó la toma de datos, bajo condiciones similares, trabajo que se realizó de manera continua, durante cinco días. Adicionalmente, antes de iniciar cada prueba de infiltración, se tomaron muestras de suelos, a una profundidad de $10 \mathrm{~cm}$, para determinar los contenidos de arena, limo y arcilla, por el método de Bouyoucos; la densidad aparente, con el método del cilindro conocido y el contenido de agua en el suelo, mediante determinación gravimétrica.

Ajuste de los modelos: Tras obtener la curva de infiltración acumulada experimental por punto de muestreo, se realizó el ajuste a los modelos empíricos de Kostiakov, KostiakovLewis y Philip. Para los dos primeros, se efectuaron gráficas logarítmicas, que transformaran las curvas de representación aritmética (originales) en líneas rectas, para someterlas a regresión estadística (mínimos cuadrados) y así determinar sus parámetros. La estimación de los parámetros del modelo de Philip (S y Ks), se ejecutó según lo descrito por Gil (2002) e implementado por Rodríguez-Vásquez et al. (2008), que consiste en una determinación implícita de la "Sortividad" (S) y la Conductividad Hidráulica Saturada (Ks), partiendo de la 
curva de infiltración acumulada experimental. Para establecer $S$ fue necesario hallar la pendiente de la curva de infiltración acumulada, correspondiente a los primeros seis minutos de la prueba. En contraste, Ks se determinó calculando la pendiente de los últimos puntos de la curva. El ajuste, se llevó a cabo mediante optimización de variables (Hyams, 2005).

Uno de los modelos más conocidos para evaluar el comportamiento de la infiltración del agua en el suelo fue desarrollado por Kostiakov (Holzapfel \& Matta, 2005; Forero, 2000):

$$
i=a * t^{b}
$$

Donde:

i Infiltración acumulada $(\mathrm{cm})$.

$\mathrm{t}$ Tiempo de infiltración en (h).

a Coeficiente el cual representa la velocidad de infiltración a $t=1$.

b Parámetro adimensional ( 0 - 1 para suelos agrícolas).

Derivando la ecuación (1) respecto al tiempo (t) y multiplicándola por un factor de conversión para llevarlo a horas, se tiene:

$$
I=\frac{d i}{d t}=60 * a * b * t^{b-1}
$$

Donde:

I Velocidad de infiltración $\left(\mathrm{cmh}^{-1}\right)$.

Para evaluar el modelo de Kostiakov es necesario estudiar la velocidad de entrada básica (infiltración básica), definida como el valor instantáneo, cuando la velocidad de cambio de entrada para un periodo estandar es $10 \%$ o menos de su valor, es decir, la infiltración se mantiene constante, a partir de cierto periodo (Holzapfel \& Matta, 2005).

$$
\frac{d I}{d t}=-0.1 * I
$$

Aplicando la ecuación (2) en (3), se tiene:

$$
\begin{aligned}
& \frac{d I}{d t}=60 * a * b *(b-1) * t^{b-2} \\
& 60 * a * b *(b-1) * t^{b-2}=-0.1 *\left(60 * a * b * t^{b-1}\right)
\end{aligned}
$$

Despejando, se obtiene:

$$
-600 *(b-1)=t \Rightarrow t_{\text {BASSICO }}
$$

La ecuación (5) representa el tiempo en el que ocurre la infiltración básica $\left(f_{0}\right)$; reemplazando el tiempo básico en la ecuación (4), se puede estimar la infiltración básica:

$$
f_{0}=\frac{d i}{d t}=60 * a * b * t_{B A S I C O}^{b-1}
$$

Una extensión del Modelo de Kostiakov es el denominado Kostiakov-Lewis. La expresión matemática, se aprecia a continuación (Castro E Souza, 1999):

$$
Z=k^{*} t^{c}+f_{0}^{*} t
$$

Donde:

Z Infiltración como una función del tiempo (cm).

$\mathrm{f}_{0} \quad$ Infiltración básica $\left(\mathrm{cm} \mathrm{min} \mathrm{m}^{-1}\right)$.

k, c Parámetros empíricos.

Derivando la ecuación (7), se obtiene la tasa de infiltración acumulada:

$$
\frac{d Z}{d t}=c^{*} k * t^{c-1}+f_{0}
$$

Donde:

dZ/dt Infiltración acumulada como una función del tiempo $\left(\mathrm{cm} \min ^{-1}\right)$.

$\mathrm{f}_{0} \quad$ Infiltración básica $\left(\mathrm{cm} \mathrm{min}{ }^{-1}\right)$.

k, c Parámetros empíricos.

Una característica de este modelo es que sus parámetros $(\mathrm{k}$, c y $f_{0}$ ) dependen, principalmente, de la textura y la estructura y del contenido de humedad del suelo, momentos previos al riego (Carvalho et al. 1999).

El tercer modelo es el de Philip, que considera la "sortividad", la cual, en términos físicos, representa la entrada de agua en el suelo por efecto del potencial matricial, consecuencia de las fuerzas de capilaridad, de adhesión y de cohesión (Osuna E Padilla, 1998) y la conductividad hidráulica saturada, que es una medida de la resistencia de los poros del suelo respecto al flujo, que intenta pasar a través de ellos (Philip, 1987):

$$
I(t)=\frac{1}{2} * S * t^{-1 / 2}+K_{S}
$$


Integrando la ecuación (9), se obtiene la lámina de infiltración acumulada:

$$
i(t)=S * t^{1 / 2}+K_{S} * t
$$

Donde:

i(t) Infiltración acumulada $(\mathrm{cm})$.

$\mathrm{t} \quad$ Tiempo de infiltración en horas.

S "Sortividad" del Suelo $\left(\mathrm{cm} \mathrm{h}^{-1 / 2}\right)$.

$\mathrm{K}_{\mathrm{S}} \quad$ Conductividad Hidráulica Saturada del Suelo

$\left(\mathrm{cm} \mathrm{h}^{-1}\right)$.

El modelo de Philip, se calcula implementando una serie de potencia, representada por una versión trucada de la serie de Taylor (Rodríguez-Vásquez et al. 2008).

En este estudio, el mejor coeficiente de determinación $\left(R^{2}\right)$ fue el criterio para determinar el modelo de mejor ajuste, verificando su significancia, mediante la prueba de hipótesis t. Adicionalmente, las propiedades del suelo y los parámetros de cada modelo, se sometieron a un análisis descriptivo, con el fin de establecer el comportamiento, la dispersión y la tendencia de los datos, a través de la media, mínimo, máximo y coeficiente de variación. Para el análisis del CV, se consideró una variabilidad baja, para $\mathrm{CV}$, menores del $12 \%$; variabilidad media, para CV, entre 12 y $60 \%$ y variabilidad alta, para valores mayores a 60\% (Warrick $\mathcal{E}$ Nielsen, 1980).

\section{RESULTADOS Y DISCUSIÓN}

De acuerdo con los resultados, la propiedad del suelo que reflejó menor variabilidad fue la densidad aparente (Tabla 1), pero con valores mayores a los reportados por Jaimes et al. (2003) y Ramírez-López et al. (2008), autores que indican que estos suelos presentan un proceso natural de adensamiento.
Los contenidos de arena, de limo y de arcilla corresponden a los rangos observados para la zona de estudio, donde la arena y el limo registraron la menor variabilidad con CV, de 11,58 y $7,06 \%$, respectivamente. De acuerdo con Rodríguez-Vásquez et al. (2008) es común que las partículas predominantes en el suelo muestren menor variabilidad. Acorde con los valores observados para los contenidos de arena, de limo y de arcilla, se verifica que las texturas predominantes del suelo en el área de estudio, se encuentran entre francas y franco arcillosas.

Los modelos que mejor ajuste presentaron fueron los de Kostikov y Kostikov-Lewis (Tabla 2), con los mayores valores del coeficiente de determinación $\left(R^{2}\right)$, aun cuando el $R^{2}$ del modelo de Philip es también adecuado, denotando una buena representatividad del modelo de los datos obtenidos en campo. En estudio realizado en suelo franco arenoso, Machiwal et al. (2006) notaron que el modelo que mejor representa la tasa de infiltración es el de Philip; sin embargo, Rodríguez-Vásquez et al. (2008) apreciaron, en un suelo franco limoso, que el modelo de Kostiakov representa mejor la tasa de infiltración, señalando limitantes del modelo de Philip, en suelos que presenten altos contenidos de humedad, donde el valor de Ks puede ser negativo y, en algunos casos, no registra ajuste a dicho modelo. Por otra parte, Varadhan $\mathcal{E}$ Williams, (1998) indican que, a diferencia del modelo de Kostoakov, el de Philip no tiende a cero para tiempos infinitos, pero representa, adecuadamente, la disminución de la velocidad de infiltración, en intervalos de tiempo finitos.

Los parámetros de los modelos exhibieron, en general, una elevada variabilidad, en algunos casos, superiores al $100 \%$, demostrando que las propiedades hidrodinámicas pueden presentar comportamientos extremos, debido a la variabilidad inherente del suelo y a que los procesos físicos latentes en el suelo no son lineales en el tiempo y en el espacio (Lima $\mathcal{E}$ Silans, 1999). Sólo los parámetros b y k mostraron variabilidad

Tabla 1. Descripción de propiedades físicas del suelo de la densidad aparente (Da), humedad gravimétrica (Hg) y los contenidos de arena, de limo y de arcilla, en la Estación Experimental Taluma.

\begin{tabular}{|c|c|c|c|c|}
\hline PROPIEDAD & UNIDAD & RANGO & MEDIA & CV (\%) \\
\hline $\mathrm{Da}$ & $\mathrm{g} \mathrm{cm}^{-3}$ & $1,564-2,072$ & 1,875 & 5,83 \\
\hline $\mathrm{Hg}$ & $\%$ & $2,143-13,013$ & 4,775 & 44,08 \\
\hline Arena & $\%$ & $23,197-45,947$ & 38,910 & 11,58 \\
\hline Limo & $\%$ & $32,214-46,679$ & 36,898 & 7,06 \\
\hline Arcilla & $\%$ & $18,727-36,540$ & 24,191 & 16,98 \\
\hline
\end{tabular}


Tabla 2. Rango de parámetros para tres modelos de infiltración, obtenidos en la Estación Experimental Taluma.

\begin{tabular}{|c|c|c|c|c|}
\hline \multirow{3}{*}{ MODELO } & PARÁMETRO MODELO & RANGO & MEDIA & CV (\%) \\
\hline \multirow{3}{*}{ Kostiakov } & $\mathrm{a}$ & $0,12-3,44$ & 0,89 & 74,28 \\
\cline { 2 - 5 } & $\mathrm{b}$ & $0,26-0,85$ & 0,54 & 26,28 \\
\cline { 2 - 5 } & $\mathrm{R}^{2}$ & $0,89-0,99$ & 0,98 & 1,67 \\
\hline \multirow{3}{*}{ Kostiakov - Lewis } & $\mathrm{c}$ & $0,05-7,11$ & 1,19 & 92,02 \\
\cline { 2 - 5 } & $\mathrm{k}$ & $0,12-0,72$ & 0,35 & 24,57 \\
\cline { 2 - 5 } & $\mathrm{fo}_{\mathrm{cm} \mathrm{h}} \mathrm{cm}^{-1}$ & $0,26-9,99$ & 1,99 & 97,74 \\
\hline \multirow{3}{*}{ Philip } & $\mathrm{R}^{2}$ & $0,90-0,99$ & 0,99 & 1,55 \\
\hline & $\mathrm{S}, \mathrm{cm} \mathrm{h}^{-1 / 2}$ & $0,540-17,294$ & 5,368 & 61,56 \\
\cline { 2 - 5 } & $\mathrm{Ks}, \mathrm{cm} \mathrm{h}^{-1}$ & $0,008-8.669$ & 1,186 & 114,02 \\
\hline & $\mathrm{R}^{2}$ & $0,68-0,99$ & 0,98 & 4,75 \\
\hline
\end{tabular}

media. La variabilidad observada, así como los valores medios y rangos encontrados, coinciden con estudios realizados por Machiwal et al. (2006) y Rodríguez-Vásquez et al. (2008).

Para todos los casos, los valores de S resultaron ser positivos; sus cuantías elevadas suponen la presencia de macroporos desarrollados por actividad biológica (Sharma et al. 1980) y demuestran que el suelo se encontraba en déficit hídrico al momento de efectuar las pruebas de infiltración, supuesto corroborado, con los datos de los contenidos de humanidad, hallados en campo. Para los valores de $\mathrm{f}_{0}$, el tiempo básico correspondiente, se aproxima a los $300 \mathrm{~min}$, demostrando que el suelo no se acercó a una condición de saturación, influyendo en la alta variabilidad para este parámetro.
El análisis de correlación indica que la variabilidad observada en los parámetros de los modelos es influenciada por la variabilidad de las propiedades del suelo, donde la Da y los contenidos de arena y de arcilla determinan el comportamiento de la velocidad de infiltración (Tabla 3).

La densidad aparente mostró una correlación inversa con la mayoría de los parámetros de los modelos, exceptuándose los parámetros a y c de los modelos Kostiakov y KostiakovLewis, respectivamente, con los cuales, se percibe las menores correlaciones. Este comportamiento confirma que a mayor densidad aparente, la velocidad de infiltración disminuye. A su vez, el contenido de humedad del suelo indica una correlación directa con el primer parámetro de todos los modelos,

Tabla 3. Coeficientes de correlación entre los parámetros de los modelos de infiltración y las propiedades físicas del suelo de la densidad aparente $(\mathrm{Da})$, humedad gravimétrica $(\mathrm{Hg})$ y los contenidos de arena, de limo y de arcilla, en la Estación Experimental Taluma.

\begin{tabular}{|c|c|c|c|c|c|c|}
\hline MODELO & PARÁMETRO & $\mathrm{Da}$ & $\mathrm{Hv}$ & ARENA & LIMO & ARCILLA \\
\hline \multirow{2}{*}{ Kostiakov } & $\mathrm{a}$ & 0,012 & 0,148 & $-0,035$ & 0,007 & 0,034 \\
\cline { 2 - 7 } & $\mathrm{b}$ & $-0,197$ & $-0,008$ & $-0,185$ & 0,099 & 0,141 \\
\hline Kostiakov-Lewis & $\mathrm{k}$ & $-0,180$ & 0,133 & $-0,186$ & 0,027 & 0,187 \\
\hline & $\mathrm{c}$ & 0,164 & 0,069 & 0,186 & $-0,039$ & $-0,179$ \\
\hline & fo & $-0,379$ & 0,025 & $-0,143$ & 0,055 & 0,123 \\
\hline & S & $-0,266$ & 0,261 & $-0,126$ & $-0,110$ & 0,209 \\
\hline Philip & Ks & $-0,190$ & $-0,087$ & $-0,019$ & 0,193 & $-0,102$ \\
\hline
\end{tabular}


denotando que su valor está estrechamente relacionado con el contenido de agua en el suelo, al momento de iniciar la prueba, como fue encontrado por Iroumé \& Schäfer (2000) y Rodríguez-Vásquez et al. (2008). De las partículas del suelo, las que mayor incidencia tienen en los parámetros de los modelos, son los contenidos de arena y de arcilla. Rodríguez-Vásquez et al. (2008) reportan un comportamiento similar de estas correlaciones, cuando el contenido de humedad del suelo es bajo. Por otra parte, estos autores encontraron que si aumenta el contenido de agua en el suelo, los parámetros de los modelos varían, presentándose cambios en las correlaciones, especialmente, las relacionadas con Da y los contenidos de arena, de limo y de arcilla.

En la figura 1, se observa el ajuste de los datos de infiltración acumulada y la tasa de infiltración del punto 43, para los tres modelos propuestos. En esta figura, se aprecia el ajuste de los datos a una curva potencial decreciente, que describe la tasa de infiltración. El fuerte cambio en los primeros instantes de la curva confirma que el suelo se hallaba en déficit hídrico en el momento de la prueba, dado que el contenido de humedad volumétrico, para el punto de muestreo, fue de 3,89\%. Dada la tendencia de las curvas, los resultados sugieren que en algunos puntos el suelo no alcanzó una condición cercana a la saturación, luego de ser sometido a una aplicación constante de agua, por un periodo de 180 minutos, especialmente, para aquellos sitios que registraron una tasa de infiltración alta. Este comportamiento fue observado para la totalidad de las pruebas de infiltración realizadas.

Las curvas de infiltración acumulada para los 64 puntos de muestreo, se señalan en la figura 2, junto con la infiltración acumulada media. La tendencia de las curvas sugiere la existencia de variabilidad espacial de las propiedades hidrofísicas, demostrando que variables como el contenido de humedad, la textura y la densidad aparente influyen en la capacidad de un suelo para retener e infiltrar agua.

Para confirmar la veracidad de los modelos, se realizó el ajuste de los datos experimentales frente a las predicciones de los modelos, para la tasa de infiltración, del punto 43 (Figura 3). Se advierte que el modelo con mejor desempeño es el de Kostikov-Lewis, seguido del modelo de Kostiakov, como fue observado con los $\mathrm{R}^{2}$ próximos de uno para estos

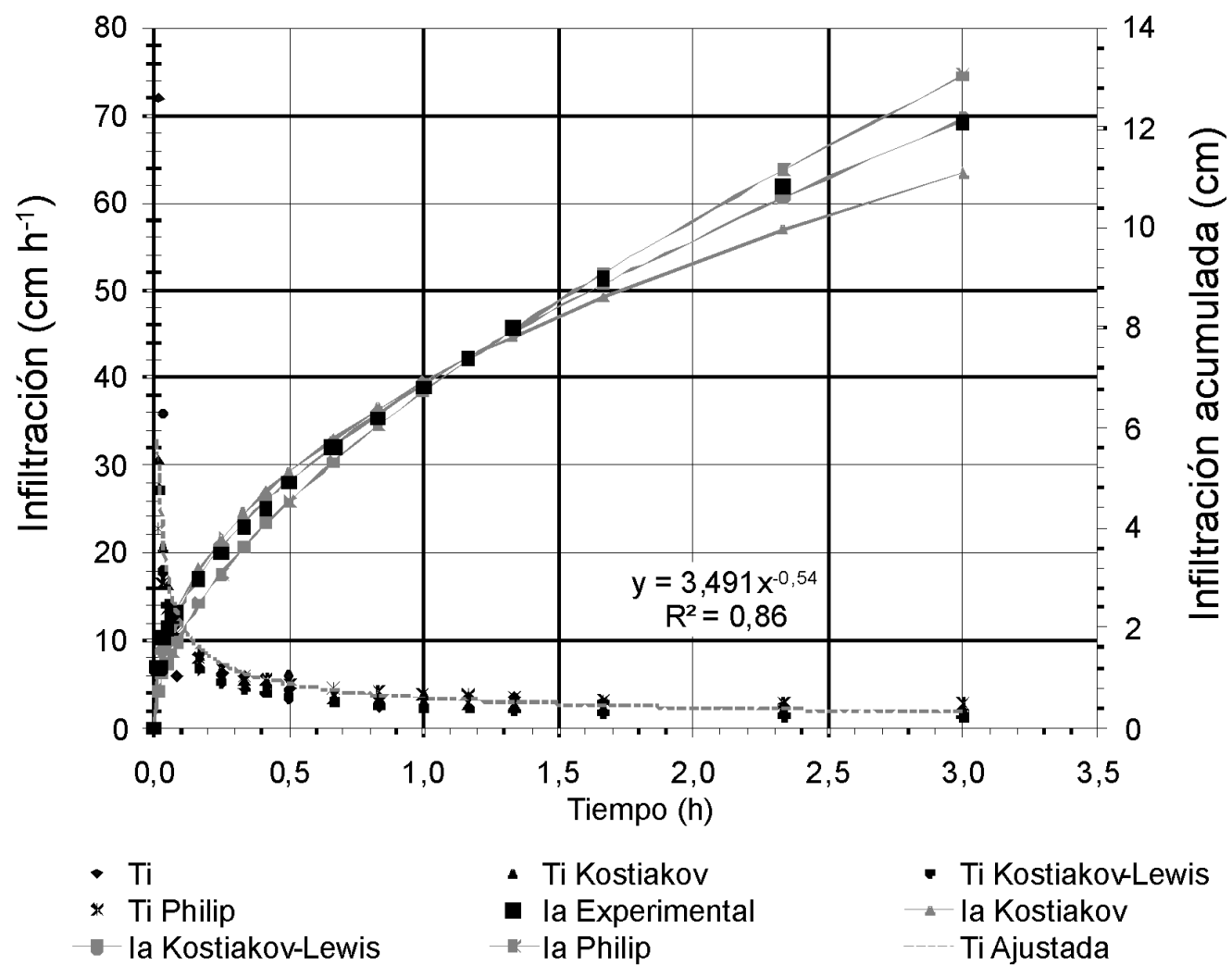

Figura 1. Ajuste de los datos de infiltración acumulada experimental a los modelos Kostiakov, Kostiakov-Lewis y Philip, en la Estación Experimental Taluma. Ti: tasa de infiltración; la: infiltración acumulada. 


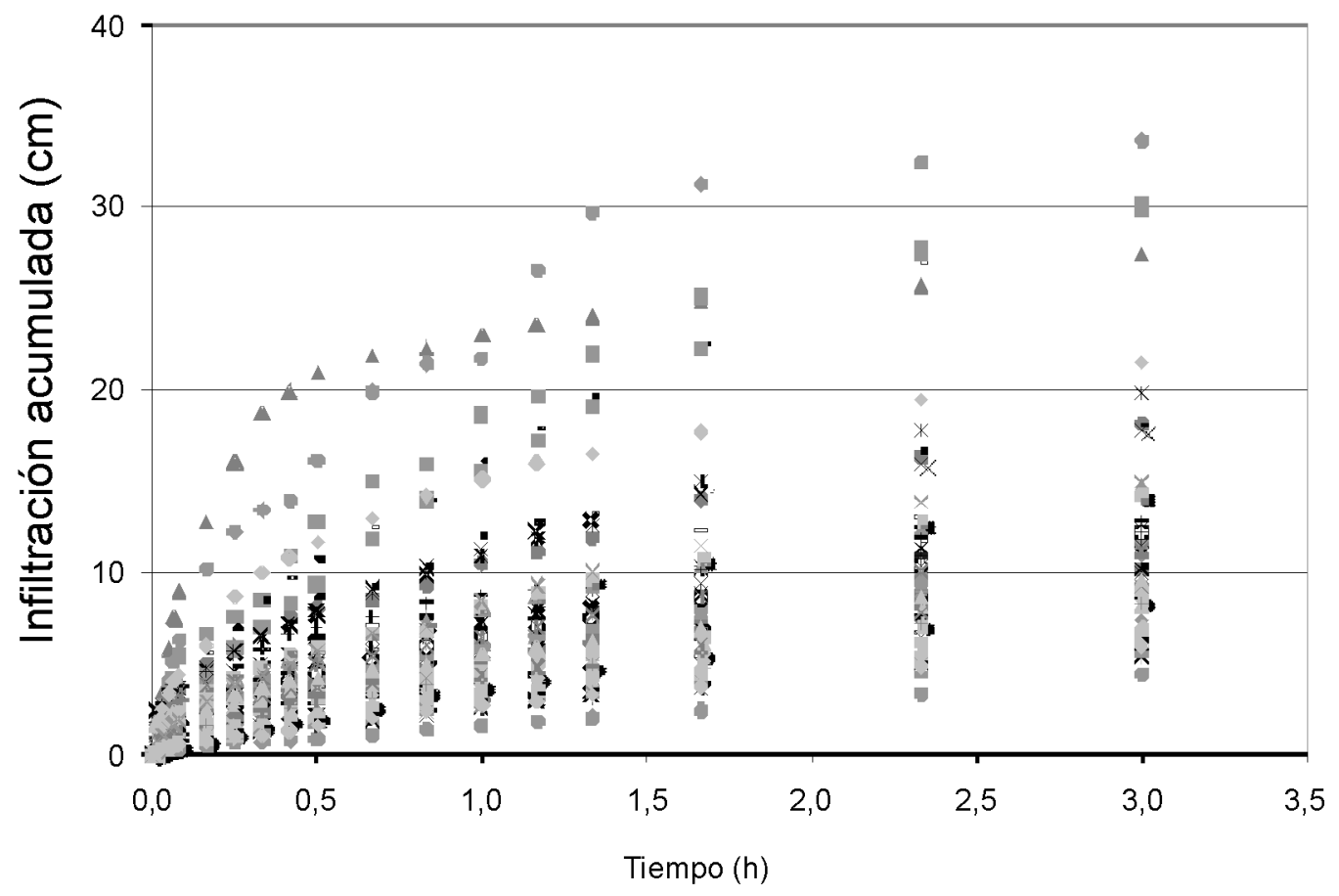

Figura 2. Curvas de infiltración acumulada e infiltración acumulada promedio para 64 puntos de muestreo, en la Estación Experimental Taluma.

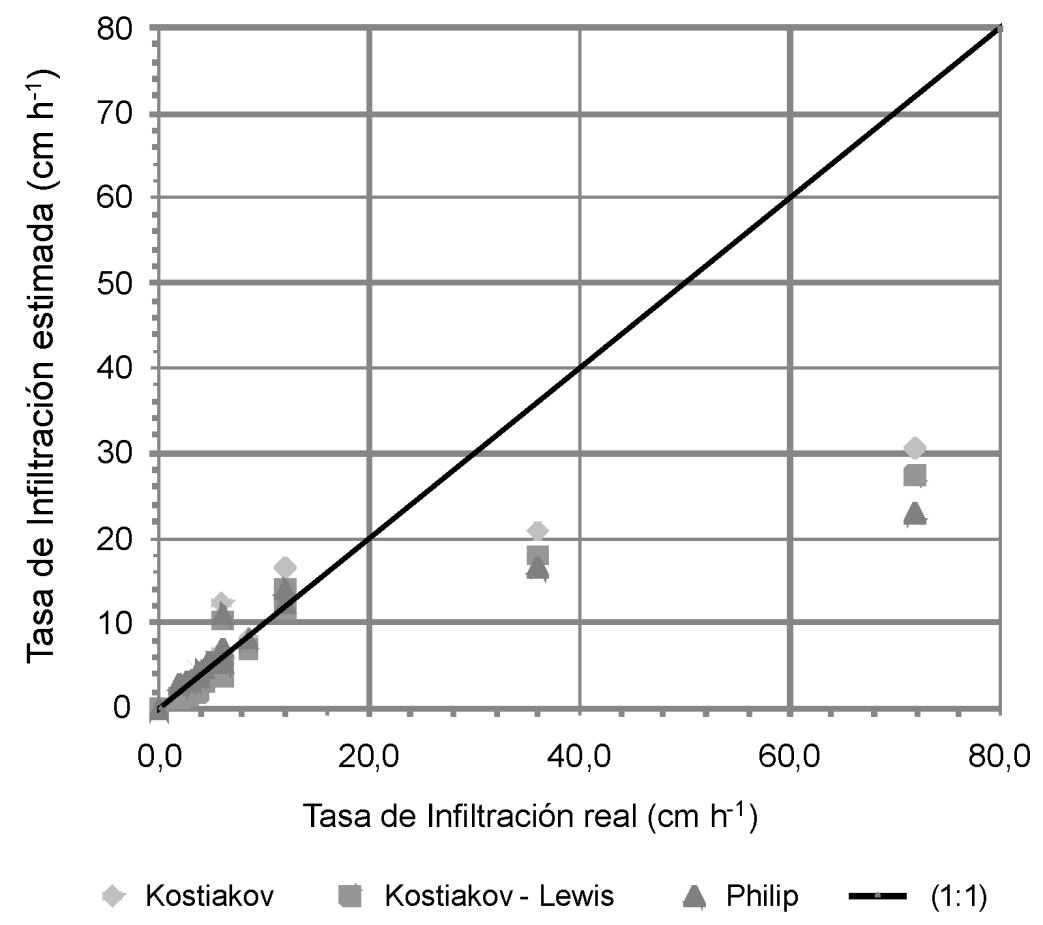

Figura 3. Ajuste de la tasa de infiltración medida frente a las estimadas con los modelos para el punto de muestreo 43 , de la Estación Experimental Taluma. 
modelos, además de los bajos CV, hallados para ambos casos. La inclusión de un parámetro adicional en el modelo de Kostiakov-Lewis, respecto del modelo de Kostiakov, sustentado por la inclusión de la infiltración básica $\left(\mathrm{f}_{0}\right)$, permite establecer el límite para evaluar la condición hasta que se hace relevante una prueba de infiltración.

Los tres modelos no estiman adecuadamente la tasa de infiltración durante los primeros minutos, pero después de 30 minutos, se descubre que los modelos de Kostiakov y Philip tienden a estimar, apropiadamente, la magnitud de la infiltración, lo que sugiere que el contenido de humedad del suelo, después de dicho periodo, se encuentra por encima del punto de marchitez permanente y cercano a capacidad de campo. Vale la pena resaltar que el anterior análisis representa los casos para los que el modelo de Kostiakov-Lewis fue la ecuación de infiltración de mejor ajuste.

Es conveniente considerar que la infiltración básica $\left(\mathrm{f}_{0}\right)$ es un criterio esencial para definir la intensidad de riego, que busca evitar aplicaciones deficientes o excesivas de agua. Por esto, el hecho de percibir una alta variabilidad de dicho parámetro, es indicio que en varios sitios el suelo no llegó a una condición cercana a la saturación, especialmente, aquellos donde la tasa de infiltración fue alta. Sin duda, esta variabilidad es definida por la heterogeneidad de las propiedades físicas y la complejidad que relacionan los diferentes atributos hidrofísicos, entre los que se puede destacar el tamaño y la forma de los agregados, la continuidad y la forma de los poros, así como la presencia y la distribución de raíces (Imhoff et al. 2010). Dadas las características físicas de la zona de estudio, la alternativa más eficiente sugerida es aumentar los tiempos de prueba de infiltración, si estas se realizan en época seca, para que el suelo se acerque a la saturación, lo que, posiblemente, pude conllevar a una disminución en la variabilidad de la infiltración básica.

Aunque los datos experimentales tuvieron un mejor ajuste al modelo de Kostiakov-Lewis, los tres modelos evaluados proporcionaron resultados adecuados, como lo demuestra la similitud de los coeficientes de determinación $\left(\mathrm{R}^{2}\right)$. Por otro lado, la variabilidad de la infiltración señala la conveniencia de identificar zonas de manejo, con el fin de evitar déficit o excesos de agua, que pueden someter el cultivo a estrés hídrico o favorecer la formación de procesos de escorrentía superficial.

Agradecimientos: A la Dirección de Investigación de la Universidad Nacional de Colombia, sede Bogotá, por el apoyo económico para el desarrollo del presente estudio. A la Corporación Colombiana de Investigación Agropecuaria (CORPOICA), por autorizar el desarrollo del presente estudio en la Estación Experimental Taluma y el apoyo en las labores de campo. Conflictos de interés: La investigación y el manuscrito se realizaron con la colaboración de todos los autores, quienes declaramos que no existe ningún conflicto de intereses que ponga en riesgo la validez de los resultados presentados.

\section{BIBLIOGRAFÍA}

1. CARVALHO, J.J.; AZEVEDO, C.A.V.; GOMES, E.M.; HENRIQUE, J.; DANTAS NETO, J. 1999. Efeito das variações espaço-temporais dos parâmetros de Kostiakov-Lewis na infiltração acumulada. Rev. Brás. Engenharia Agrícola e Ambiental. 3(3):268-275.

2. CASTRO, L.C.A.; SOUZA, F. 1999. Análise dos parâmetros da equação de infiltração de Kostiakov-Lewis na irrigação por sulcos. Rev. Brás. Engenharia Agrícola e Ambiental. 3(2):167-172.

3. CHOWDARY, V.M.; DAMODHARA RAO, M.; JAISWAL, C.S. 2006. Study of infiltration process under different experimental conditions. Agr. Water Management. 83(1-2):69-78.

4. DIAMOND, J.; SHANLEY, T. 2003. Infiltration rate assessment of some major soils. Irish Geography. 36(1):32-46.

5. FORERO, J.A. 2000. Parámetros Hidrodinámicos para Riego. Universidad Nacional de Colombia. Facultad de Ingeniería. Unidad de Publicaciones. 31p.

6. GIL, R. 2002. El comportamiento físico-funcional de los suelos. Instituto de Suelos INTA Castelar.19p.

7. GREEN, W.H.; AMPT, G.A. 1911. Studies on Soil PhysicsPart I: the flow of air and water through soils. J. Agr. Science. 4(1):1-24.

8. HAVERKAMP, R.; BOURAOUI, F.; ZAMMIT, C.; ANGULOJARAMILLO, R. 1998. Soil properties and moisture movement in the unsaturated zone. In: Delleur, J.W. (Ed.). Groundwater Engineering Handbook. CRC Press, Boca Raton, FL. p.5.1-5.50.

9. HOLZAPFEL, E.A.; MATTA, C.R. 2005. Infiltración de agua en el suelo. Universidad de Concepción. Facultad de Ingeniería Agrícola. Departamento de Recursos Hídricos. 30p.

10. HORTON, R.E. 1940. An approach toward a physical interpretation of infiltration-capacity, Soil Science Soc. Am. Proc. 5:399-417. 
11. HYAMS, D. 2005. Curve expert versión 1.4. A comprehensive curve fitting package for Windows. [Online], http://curveexpert.webhop.biz/ (con acceso 05/01/10).

12. IMHOFF, S.; GHIBERTO, P.J.; GRIONI, A.G.; GAY, J.P. 2010. Porosity characterization of Argiudolls under different management systems in the Argentine Flat Pampa. Geoderma. 158(3-4):268-274.

13. IROUMÉ, A.; SCHÄFER, M. 2000. Variabilidad espacial y temporal de la infiltración en una cuenca experimental en la cordillera de los Andes, IX Región, Chile. Agro sur. 28(2):1-9.

14. JAIMES, W.; NAVAS, G.; SALAMANCA, C.R.; CONDE, A. 2003. Estudio detallado de suelos de la Estación Experimental Sabanas en la altillanura colombiana. Corpoica, C.I. La Libertad, Villavicencio, Colombia. 62p.

15. KOSTIAKOV, A.N. 1932. On the dynamics of the coefficient of water-percolation in soils and on the necessity of studying it from a dynamic point of view for purposes of amelioration, Transactions Congress International Society for Soil Science, 6th, Moscow Part A, p.17-21.

16. LIMA, C.A.G.; SILANS, A.P de. 1999. Variabilidade espacial da infiltração de água no solo. Pesquisa Agropecuária Brasileira. 34(12):2311-2320.

17. LOAGUE, K.; GANDER, G.A. 1990. R-5 Revisited 1. Spatial Variability of Infiltration on a Small Rangeland Catchment. Water Resources Res. 26(5):957-971.

18. MACHIWAL, D.; JHA, M.; MAL, B.C. 2006. Modelling infiltration and quantifying spatial soil variability in a wasteland of Kharagpur, India. Biosystems Engineering. 95(4):569-582.

19. OSUNA CEJA, E.S.; PADILLA RAMÍREZ, J.S. 1998. Estimación de la sorbilidad e infiltración usando datos de simulación de lluvia para tres tipos de suelos de la zona semiárida de México. Terra. 16(4):293-302.
20. PHILIP, J.R. 1957. The theory of infiltration: 4. Sorptivity and algebraic infiltration equations. Soil Science. 84:257-264.

21. PHILIP, J.R. 1987. The infiltration joining problem. Water Res. 23:2239-2245.

22. RAMÍREZ-LÓPEZ, L.; REINA-SÁNCHEZ, A.; CAMACHOTAMAYO, J.H. 2008. Variabilidad espacial de atributos físicos de un Typic Haplustox de los Llanos Orientales de Colombia. Eng. Agric. 28(1):55-63.

23. RODRÍGUEZ-VÁSQUEZ, A.F.; ARISTIZÁBAL-CASTILLO, A.M.; CAMACHO-TAMAYO, J.H. 2008. Variabilidad espacial de los modelos de infiltración de Philip y Kostiakov en un suelo Ándico. Eng. Agric. 28(1):64-75.

24. SHARMA, M.L.; GANDER, G.A.; HUNT, C.G. 1980. Spatial variability of infiltration in a watershed. J. Hydrology. 45(1-2):101-122.

25. SILVA, L.L. 2007. Fitting infiltration equations to centrepivot irrigation data in a mediterranean soil. Agr. Water Management. 94(1-3):83-92.

26. UNITED STATES DEPARTMENT OF AGRICULTURE. 1999. Soil Taxonomy. Second Edition. Agriculture Handbook. No. 436.

27. VARADHAN, R.; WILLIAMS, J.R. 1998. Estimation of infiltration rate in the vadose zone: compilation of simple material models: application of selected mathematical models. Ada: National risk management Research laboratory, U.S. EPA. v.1, 84p. (EPA/600/R-97/128b).

28. WARRICK, A.W.; NIELSEN, D.R. 1980. Spatial variability of soil physical properties in the field. En: Hillel, D. (Ed.). Applications of soil physics. New York: Academic Press, p.319-344.

Recibido: Abril 23 de 2010

Aceptado: Septiembre 6 de 2010 